\title{
ISOMETRIC IMMERSIONS IN THE HYPERBOLIC SPACE WITH THEIR IMAGE CONTAINED IN A HOROBALL
}

\author{
ANA LLUCH ${ }^{\dagger}$ \\ Departamento de Mathematicas, Universitat Jaume I, \\ 12080 Castellon de la Plana, Spain
}

(Received 18 February, 1999)

\begin{abstract}
We give a sharp lower bound for the supremum of the norm of the mean curvature of an isometric immersion of a complete Riemannian manifold with scalar curvature bounded from below into a horoball of a complex or real hyperbolic space. We also characterize the horospheres of the real or complex hyperbolic spaces as the only isometrically immersed hypersurfaces which are between two parallel horospheres, have the norm of the mean curvature vector bounded by the above sharp bound and have some special groups of symmetries.
\end{abstract}

1991 Mathematics Subject Classification. 53C42, 53C21.

1. Introduction. A classical problem in submanifold theory is the existence of complete non planar minimal surfaces in some "bounded" set in $\mathbb{R}^{3}$. A breakthrough in this problem was the construction, by Jorge and Xavier (see [10]), of non planar, minimal, isometric immersions of complete surfaces between two parallel planes in $\mathbb{R}^{3}$. The same authors [11] and Koutroufiotis [9] showed that if the immersed manifold has scalar curvature bounded from below, then there is no minimal immersion inside a ball.

Another negative result was given by Hoffman and Meeks in [7]. They proved that the only properly immersed minimal surfaces in $\mathbb{R}^{3}$ which are contained in a halfspace are the planes parallel to the boundary of the halfspace. They show also that this theorem is not true for higher dimensions.

An analogous problem for the hyperbolic space $\mathbb{H}^{n}(\lambda)$ of constant sectional curvature $\lambda<0$, is the existence of minimal immersions (or, with more generality, immersions of constant mean curvature) with their images contained in a horoball.

In this paper, we consider this problem for immersed submanifolds from the point of view of [9] and [11], and we shall prove the following result.

Theorem 1. Let $\psi: M \rightarrow \mathbb{H}^{n}(\lambda)$ be an isometric immersion of a complete Riemannian manifold $M$ of dimension $n-1$ with scalar curvature bounded from below. If $\psi(M) \subset \mathcal{H B}$ for some horoball $\mathcal{H B}$ of $\mathbb{T}^{n}(\lambda)$, then

$$
\sup _{x \in M}\|H\| \geq \sqrt{|\lambda|}
$$

where $H$ denotes the mean curvature vector of the immersion, and sup denotes the supremum.

${ }^{\dagger}$ Work partially supported by DGICYT Grant No. PB97-1425. 
As a consequence we have that if $M$ is an $(n-1)$-dimensional connected and complete Riemannian manifold with scalar curvature bounded from below, then there is no isometric immersion of $M$ in $\mathbb{T}^{n}(\lambda)$ contained in a horoball and with $\|H\| \leq c<\sqrt{|\lambda|}$.

Do Carmo and Lawson [4] proved that the only embedded hypersurfaces in the hyperbolic space with constant mean curvature and only one point in the infinite boundary of $\mathbb{H}^{n}(\lambda)$ are the horospheres. Since any complete non-compact embedded submanifold contained in an horoball has only one point in the infinite boundary of $\mathbb{H}^{n}(\lambda)$, this result solves the problem quoted before about immersions contained in horoballs for embeddings. But Do Carmo and Lawson say that the assumption of embeddedness in their result is necessary because of some examples given by Gomes in [5].

If, in the above theorem by Do Carmo and Lawson, we change the hypothesis "constant mean curvature" to " $\|H\| \leq \sqrt{|\lambda|}$ ", then there are many embeddings with only one point in the infinite boundary of $\mathbb{H}^{n}(\lambda)$. A simple example is the hypersurface of $\mathbb{H}^{3}(-1)$ given (in the Poincaré's upper halfspace model of $\mathbb{H}^{3}(-1)$ ) by the parametrization $x(u, v)=\left(u, v, e^{v}\right)$. For this surface the mean curvature is $\|H\|=\left(2+3 e^{2 v}\right) /\left(2\left(1+e^{2 v}\right)^{3 / 2}\right)<1$. However, we have not found any example of a proper immersion in $\mathbb{H}^{n}(\lambda)$ with $\|H\| \leq \sqrt{|\lambda|}$ and with its image contained in a horoball. Here we will characterize the horospheres as the unique properly immersed hypersurfaces contained between two parallel horospheres, having these symmetries and with $\|H\| \leq \sqrt{|\lambda|}$ and having some special group of symmetries.

The "group of symmetries" of an immersion $\psi: M \rightarrow \mathbb{H}^{n}(\lambda)$ is usually defined by people working on minimal immersions (see $[\mathbf{8 , 1 2}]$ ) as the restriction to $M$ of the group of isometries of $\mathfrak{H}^{n}(\lambda)$ preserving $\psi(M)$. The meaning of this definition is clear when $\psi$ is an embedding. For the convenience of the reader, we shall give the details of the definitions for general immersions.

Given any isometric immersion $\psi: M \rightarrow \mathbb{H}^{n}(\lambda)$, the group of symmetries $\operatorname{Sym}(M)$ of this immersion is the group

$$
\operatorname{Sym}(M)=\left\{A \in \operatorname{Iso}(M): \text { there is some } \alpha \in \operatorname{Iso}\left(\mathbb{M}^{n}(\lambda)\right) \text { such that } \psi \circ A=\alpha \circ \psi\right\},
$$

where $\operatorname{Iso}(M)$ and Iso $\left(\mathbb{H}^{n}(\lambda)\right)$ denote, respectively, the group of isometries of $M$ and $\mathbb{H}^{n}(\lambda)$.

When $\psi$ is a proper embedding we may speak of the isomorphism of groups

$$
\operatorname{Sym}(M) \rightarrow \operatorname{Iso}_{M}\left(\mathbb{H}^{n}(\lambda)\right) \text { defined by } A \mapsto \psi \circ A \circ \psi^{-1}
$$

which takes any subgroup $G$ of $\operatorname{Sym}(M)$ onto a subgroup $\mathcal{G}$ of $\operatorname{Iso}_{M}\left(\mathbb{H}^{n}(\lambda)\right)$. When $\psi$ is not an embedding, we do not have the above isomorphism, but we can still associate to any subgroup $G$ of $\operatorname{Sym}(M)$ a subgroup $\mathcal{G}$ of $\operatorname{Iso}\left(\mathbb{M}^{n}(\lambda)\right)$ defined by

$$
\mathcal{G}=\left\{\alpha \in \operatorname{Iso}\left(\mathbb{H}^{n}(\lambda)\right) \text { such that there is some } A \in G \text { satisfying } \psi \circ A=\alpha \circ \psi\right\} \text {. }
$$
$M$.

In the language of the definitions in [12] and [8], $G$ will be the restriction of $\mathcal{G}$ to

Moreover we shall say that two hypersurfaces of a Riemannian manifold are parallel if the points of one of them are at a constant distance from the other.

With these definitions we have the following result. 
Theorem 2. Let $\psi: M \rightarrow \mathbb{U}^{n}(\lambda)$ be a proper isometric immersion of a connected and complete $(n-1)$-dimensional Riemannian manifold $M$ satisfying:

(a) $\psi(M) \subset \mathcal{D}=\mathcal{H B}_{1}-\mathcal{H B}_{2}$, where $\mathcal{H} \mathcal{B}_{i}$ is an horoball having the horosphere $\mathcal{H}_{i}$ as boundary and $\mathcal{H}_{1}$ and $\mathcal{H}_{2}$ are parallel horospheres,

(b) $\|H\| \leq \sqrt{|\lambda|}$, and

(c) there is a subgroup $G$ of $\operatorname{Sym}(M)$ such that $\mathcal{D}$ is invariant under a properly discontinuous action of $\mathcal{G}$ and the quotient manifold $\mathcal{D} / \mathcal{G}$ is compact.

Then $\psi(M)$ is a horosphere and $\psi$ is an embedding.

We would like to remark that, in some sense, we may see the hypersurfaces in $\mathbb{H}^{n}(\lambda)$ with $\|H\| \leq \sqrt{|\lambda|}$ as the objects analogous to minimal hypersurfaces in $\mathbb{R}^{n}$. In fact, in [11] and [9], to prove the above mentioned result of non-immersibility, the authors give the following more general result: "If $M$ is a complete Riemannian manifold with scalar curvature bounded from below, there is no isometric immersion of $M$ into a space of constant sectional curvature $\mu \leq 0$, having mean curvature $\|H\| \leq \sqrt{|\mu|}$ and with its image contained in a ball". When $\mu=0$, we have the nonimmersibility theorem for minimal submanifolds in $\mathbb{R}^{n}$ contained in a ball (and we have also that there are no immersed compact hypersurfaces with $\|H\| \leq \sqrt{|\mu|}$. Then, relative to this property, minimal hypersurfaces in $\mathbb{R}^{n}$ and hypersurfaces in $\mathbb{Q}^{n}(\lambda)$ with $\|H\| \leq \sqrt{|\lambda|}$ have the same behaviour.

For the complex case, $\mathbb{H}_{\mathbb{C}}^{n}(\lambda)$ will denote the complex hyperbolic space of constant holomorophic sectional curvature $4 \lambda<0, \mathcal{H}_{\mathbb{C}}$ will denote a horosphere of $\mathbb{H}_{\mathbb{C}}^{n}(\lambda)$, which has constant mean curvature $\frac{2 n}{2 n-1} \sqrt{|\lambda|}$, and $\mathcal{H} \mathcal{B}_{\mathbb{C}}$ will denote the horoball having $\mathcal{H}_{\mathbb{C}}$ as its boundary. With the same notation as in the real case, but changing the group Iso $\left(\mathbb{H}^{n}(\lambda)\right)$ of the isometries of $\mathbb{M}^{n}(\lambda)$ to the group $\operatorname{Iso}_{H}\left(\mathbb{M}_{\mathbb{C}}^{n}(\lambda)\right)$ of the holomorphic isometries of $\mathbb{U}_{\mathbb{C}}^{n}(\lambda)$, we shall prove the following.

TheOREM 3. Let $\psi: M \rightarrow \mathbb{H}_{\mathbb{C}}^{n}(\lambda)$ be an isometric immersion of a connected and complete Riemannian manifold of dimension $2 n-1$.

(i) If $\psi(M) \subset \mathcal{H} \mathcal{B}_{\mathbb{C}}$ and $M$ has scalar curvature bounded from below, then

$$
\sup _{x \in M}\|H\| \geq \frac{2 n}{2 n-1} \sqrt{|\lambda|} .
$$

(ii) Let $\mathcal{D}_{\mathbb{C}}$ be the domain in $\mathbb{\boxplus}^{n}(\lambda)$ defined by $\mathcal{D}_{\mathbb{C}}=\mathcal{H} \mathcal{B}_{\mathbb{C}}^{1}-\mathcal{H} \mathcal{B}_{\mathbb{C}}^{2}$, where $\mathcal{H} \mathcal{B}_{\mathbb{C}}^{i}$ is a horoball with boundary the horosphere $\mathcal{H}_{\mathbb{C}}^{i}$, and $\mathcal{H}_{\mathbb{C}}^{1}$ and $\mathcal{H}_{\mathbb{C}}^{2}$ are parallel horospheres. If $\psi$ is proper, $\psi(M) \subset \mathcal{D}_{\mathbb{C}},\|H\| \leq \frac{2 n}{2 n-1} \sqrt{|\lambda|}$, and there is a subgroup $G$ of $\operatorname{Sym}(M)$ such that $\mathcal{G}\left(\mathcal{D}_{\mathbb{C}}\right)=\mathcal{D}_{\mathbb{C}}, \mathcal{G}\left(\mathcal{H}_{\mathbb{C}}^{i}\right)=\mathcal{H}_{\mathbb{C}}^{i}$, the action of $\mathcal{G}$ on $\mathcal{D}_{\mathbb{C}}$ is properly discontinuous and the quotient manifold $\mathcal{D}_{\mathbb{C}} / \mathcal{G}$ is compact, then $\psi(M)$ is a horosphere and $\psi$ is an embedding.

I wish to express my gratitude to V. Miquel for his careful proofreading and many useful suggestions, and to V. Cervera and V. Palmer for some useful talks.

2. Notation and preliminary results. Let $\psi: M \rightarrow \mathbb{H}^{n}(\lambda)$ be an isometric immersion of a hypersurface, such that $\psi(M) \subset \mathcal{H B}$. Let $r: \mathbb{M}^{n}(\lambda) \rightarrow \mathbb{R}$ be the distance (in $\mathbb{H}^{n}(\lambda)$ ) to the horosphere $\mathcal{H}$ which is the boundary of $\mathcal{H} \mathcal{B}$, and we denote 
also by $r$ the composition $r \circ \psi$. Let us denote by $\partial_{r}$ the gradient of $r$ in $\mathbb{H}^{n}(\lambda)$ and by $\partial_{r}^{\top}$ the vector field on $M$ defined by $\partial_{r}^{\top}(q)=\psi_{*}^{-1}\left(\mathcal{P}_{q}\left(\partial_{r}(\psi(q))\right)\right.$ for every $q \in M$, where $\mathcal{P}_{q}$ denotes the orthogonal projection $\mathcal{P}_{q}: T_{\psi(q)} \mathbb{H}^{n}(\lambda) \rightarrow \psi_{*} T_{q} M$.

We denote by $S(r)$ the $(1,1)$-tensor field on $\mathbb{H}^{n}(\lambda)$ defined by:

$$
S(r)(A)=-\bar{\nabla}_{A} \partial_{r}
$$

where $\bar{\nabla}$ denotes the covariant derivative on $\mathbb{H}^{n}(\lambda)$. Let us observe that $S(r) \partial_{r}=0$, and $S(r)$ restricted to the subspace $\left\{\partial_{r}\right\}^{\perp}$ of the tangent space to $\mathbb{H}^{n}(\lambda)$ is the Weingarten map (associated to the unit normal vector $\partial_{r}$ ) of the tubular hypersurface of radius $r$ around the horosphere $\mathcal{H}$, and $\operatorname{tr} S(r)$ is $(n-1)$ times the mean curvature of this tubular hypersurface. It is known that the tubular hypersurface of a horosphere in $\mathbb{H}^{n}(\lambda)$ is again an horosphere; then we have (cf., for instance, [3, p. 184]) that

$$
S(r)(X)=\sqrt{|\lambda|} X \text { for all } X \in\left\{\partial_{r}\right\}^{\perp} .
$$

From now on, $\nabla, \Delta$ and $H$ will denote, respectively, the covariant derivative of $M$, the laplacian of $M$, and the mean curvature vector of the immersion. If $f: \mathbb{R} \rightarrow \mathbb{R}$ is any function, $f(r)$ will denote the composition $f \circ r$. Then (see, for instance, [2]), we have the following formula:

$$
\Delta f(r)=-f^{\prime \prime}(r)\left\|\partial_{r}^{\top}\right\|^{2}+f^{\prime}(r)\left\{\sum_{i=1}^{n-1}<S(r) e_{i}, e_{i}>-(n-1)<H, \partial_{r}>\right\}
$$

where $\left\{e_{i}\right\}_{i=1}^{n-1}$ is a local orthonormal frame of vector fields tangent to $M, S(r) e_{i}$ means $S(r) \psi_{*}\left(e_{i}\right)$, and $<,>$ is the metric on $\mathbb{H}^{n}(\lambda)$.

We shall use the following lemmas.

OMORI'S LEMMA [13]. Let $M$ be a complete Riemannian manifold whose sectional curvature is bounded from below. Let $g: M \rightarrow \mathbb{R}$ be a smooth function bounded from above. Then, for every $p \in M$ and every $\varepsilon>0$, there is a point $p^{\prime} \in M$ with the properties:

$$
g\left(p^{\prime}\right) \geq g(p), \quad\|\operatorname{grad} g\|\left(p^{\prime}\right)<\varepsilon, \quad \nabla^{2} g(X, X)<\varepsilon\|X\|^{2}
$$

for every $X \in T_{p^{\prime}} M$.

The next lemma follows in the same way as the Lemma of theorem $\mathrm{H}$ in [9], taking into account the fact that the ambient manifold is of constant sectional curvature.

Lemma 1. Let $\psi: M \rightarrow \mathbb{H}^{n}(\lambda)$ be an isometric immersion of a complete Riemannian manifold of dimension $n-1$ with the scalar curvature bounded from below. If $\|H\| \leq H_{0}$, for some constant $H_{0}>0$, then the sectional curvature of $M$ is bounded in absolute value. 


\section{Proof of the results.}

Proof of Theorem 1. Under the hypothesis of Theorem 1.1, using the equations (2.2) and (2.3),

$$
\begin{aligned}
\Delta f(r)= & -f^{\prime \prime}(r)\left\|\partial_{r}^{\top}\right\|^{2}+f^{\prime}(r)\left\{\sum_{i=1}^{n-1}<S(r) e_{i}, e_{i}>-(n-1)<H, \partial_{r}>\right\} \\
= & -f^{\prime \prime}(r)\left\|\partial_{r}^{\top}\right\|^{2}+f^{\prime}(r)\left\{\sum_{i=1}^{n-1}<S(r)\left(e_{i}-<e_{i}, \partial_{r}>\partial_{r}+<e_{i}, \partial_{r}>\partial_{r}\right), e_{i}>\right. \\
& \left.-(n-1)<H, \partial_{r}>\right\} \\
= & -f^{\prime \prime}(r)\left\|\partial_{r}^{\top}\right\|^{2}+f^{\prime}(r)\left\{(n-1) \sqrt{|\lambda|}-\sqrt{|\lambda|}\left\|\partial_{r}^{\top}\right\|^{2}-(n-1)<H, \partial_{r}>\right\} \\
= & -\left(f^{\prime \prime}(r)+\sqrt{|\lambda|} f^{\prime}(r)\right)\left\|\partial_{r}^{\top}\right\|^{2}+(n-1) f^{\prime}(r)\left(\sqrt{|\lambda|}-<H, \partial_{r}>\right) .
\end{aligned}
$$

Now, if we take as function $f$ the solution $f(r)=\frac{1}{\sqrt{|\lambda|}} e^{-\sqrt{|\lambda|} r}$ of the differential equation

$$
f^{\prime \prime}(r)+\sqrt{|\lambda|} f^{\prime}(r)=0
$$

taking account of $\left|<H, \partial_{r}>\right| \leq\|H\|$, we get from (3.1) that

$$
\begin{aligned}
\Delta f(r) & =-(n-1) e^{-\sqrt{|\lambda| r}}\left(\sqrt{|\lambda|}-<H, \partial_{r}>\right) \\
& \leq-(n-1) e^{-\sqrt{|\lambda| r}}(\sqrt{|\lambda|}-\|H\|) .
\end{aligned}
$$

If there is some point $p \in M$ such that $\sqrt{|\lambda|}-\|H\| \leq 0$, then the theorem is obvious. On the contrary, Lemma 1 allows us to apply Omori's lemma to the function $g(m)=f(r(m))=\frac{1}{\sqrt{|\lambda|}} e^{-\sqrt{|\lambda|} r(m)}$; then we have that for every $p \in M$ and every $\varepsilon>0$ there is a point $p^{\prime}$ in $M$ such that

$$
\frac{1}{\sqrt{|\lambda|}} e^{-\sqrt{|\lambda| r\left(p^{\prime}\right)}} \geq \frac{1}{\sqrt{|\lambda|}} e^{-\sqrt{|\lambda| r(p)}}
$$

and

$$
\Delta f\left(r\left(p^{\prime}\right)\right)=-\sum_{i=1}^{n-1} \nabla^{2} f\left(r\left(p^{\prime}\right)\right)\left(e_{i}, e_{i}\right)>-\varepsilon(n-1) .
$$

Inequality (3.4) is equivalent to the inequality

$$
0 \geq C=-e^{-\sqrt{|\lambda| r(p)}} \geq-e^{-\sqrt{|\lambda| r\left(p^{\prime}\right)} .}
$$

By combining this with (3.3) and (3.5) we have that, for every $\varepsilon>0$,

$$
C(n-1)(\sqrt{|\lambda|}-\|H\|) \geq-e^{-\sqrt{|\lambda| r} r\left(p^{\prime}\right)}(\sqrt{|\lambda|}-\|H\|)>-\varepsilon(n-1) .
$$


Then

$$
C(n-1) \sup _{x \in M}(\sqrt{|\lambda|}-\|H\|) \geq 0,
$$

i.e.

$$
\sup _{x \in M}(\|H\|) \geq \sqrt{|\lambda|}
$$

Proof of Theorem 2. From the definitions it is easy to see that if the action of $\mathcal{G}$ on $\mathcal{D}$ is properly discontinuous, then so is the action of $G$ on $M$. Then $M / G$ and $\mathcal{D} / \mathcal{G}$ are manifolds and the projection maps $\pi$ into the quotient manifolds $M / G$ and $\mathcal{D} / \mathcal{G}$, respectively, are covering maps. These facts allow us to define on $M / G$ and $\mathcal{D} / \mathcal{G}$ the metrics induced by $\pi$. Then

$$
\bar{\psi}: M / G \rightarrow \mathcal{D} / \mathcal{G}
$$

defined as $\bar{\psi} \circ \pi=\pi \circ \psi$ is a well defined isometric immersion. If we take for $\mathbb{H}^{n}(\lambda)$ Poincaré's upper half-space model, the isometries of the hyperbolic space are the conformal maps of $\mathbb{R}^{n}$ preserving the upper half-space. These isometries, by Liouvilles's theorem, are the composition of an isometry, $i$, of $\mathbb{R}^{n}$, a dilatation, $d$, and an inversion, $I$, at point in $x_{n}=0$, and at most one of these.

Let $\alpha \in \mathcal{G}$. Then $\alpha$ is a diffeomorphism and $\alpha(\partial \mathcal{D})=\partial \mathcal{D}$, so $\alpha\left(\mathcal{H}_{1} \cup \mathcal{H}_{2}\right)=$ $\mathcal{H}_{1} \cup \mathcal{H}_{2}$. If $\alpha\left(\mathcal{H}_{1}\right)=\mathcal{H}_{2}$, from the above classification of the isometries of $\mathbb{T}^{n}(\lambda)$, there is no inversion in the expression of $\alpha$ because the isometries of $\mathbb{R}^{n}$ preserving the upper half-space and the dilatations take hyperplanes onto hyperplanes, and an inversion at a point in $x_{n}=0$ take hyperplanes in spheres. Then $\alpha=i \circ d$ or $\alpha=d \circ i$. Since $i\left(\mathcal{H}_{i}\right)=\mathcal{H}_{i}$, the only possibility for the equality $\alpha\left(\mathcal{H}_{1}\right)=\mathcal{H}_{2}$ is that $d\left(\mathcal{H}_{1}\right)=\mathcal{H}_{2}$, but this will imply $\alpha\left(\mathcal{H}_{2}\right)=d\left(\mathcal{H}_{1}\right) \subset \mathcal{D}$, which is a contradiction. Then $\alpha\left(\mathcal{H}_{1}\right)=\mathcal{H}_{1}$ and $\alpha\left(\mathcal{H}_{2}\right)=\mathcal{H}_{2}$ and we have that $\mathcal{G}\left(\mathcal{H}_{1}\right)=\mathcal{H}_{1}$ and $\pi\left(\mathcal{H}_{1}\right)=\mathcal{H}_{1} / \mathcal{G}$. Since $\pi$ is a local isometry, if we denote also by $r, \partial_{r}$ and $S(r)$ the analogs for $\bar{\psi}: M / G \rightarrow \mathcal{D} / \mathcal{G}$ of the objects defined for $\psi: M \rightarrow \mathcal{H}^{n}(\lambda)$, changing $\mathcal{H}_{1}$ to $\mathcal{H}_{1} / \mathcal{G}$, we have that the expressions for $S(r)$ and the formula (3.3) also hold in this case.

If the mean curvature of the immersion $\psi$ is bounded from above by $\sqrt{|\lambda|}$, the same is true for the immersion $\bar{\psi}$, and from (3.3) it follows that

$$
\Delta f(r) \leq 0
$$

Since $\pi: \mathcal{D} \rightarrow \mathcal{D} / \mathcal{G}$ is a Riemannian covering and $\mathcal{D} / \mathcal{G}$ is compact, there is a compact set $\mathcal{K} \subset \mathcal{D}$ such that $\pi(\mathcal{K})=\pi(\mathcal{D})$. As $\psi$ is proper map, $\psi^{-1}(\mathcal{K})$ is compact. Let $m \in M$, then we have that $\pi(\psi(m)) \in \mathcal{D} / \mathcal{G}=\pi(\mathcal{K})$, so there is some $\alpha \in \mathcal{G}$ and some $k \in \mathcal{K}$ satisfying $\alpha(\psi(m))=k$. By definition, there is some $A \in G$ such that $\psi(A(m))=\alpha(\psi(m))=k$; then $A(m) \in \psi^{-1}(\mathcal{K})$. This fact implies that $M / G=$ $\pi\left(\psi^{-1}(\mathcal{K})\right)$. Since $\psi^{-1}(\mathcal{K})$ is compact and $\pi$ is continuous, $M / G$ is compact. Then, by the Stokes' Theorem, $0=\int_{M / G} \Delta f(r)$, and from (3.6) we will have $\Delta f(r)=0$. Then all the inequalities we have used to get (3.6) must be equalities. This will imply that $r$ has to be constant and $\|H\|=\sqrt{|\lambda|}$. So $\bar{\psi}(M / G)$ must be a tubular hypersurface of constant radius around $\mathcal{H}_{1} / \mathcal{G}$. Then $\psi(M)$ is contained in a horosphere $\mathcal{H}$ at constant distance from $\mathcal{H}_{1}$. Since $M$ and $\mathcal{H}$ have the same dimension, $M$ is complete and $\psi$ is a local isometry, so $\psi(M)=\mathcal{H}$ and $\psi$ is a covering map (see [3, p. 150]). Then $\psi$ is a diffeomorphism, since $\mathcal{H}$ is simply connected. 
Proof of Theorem 3. (i). Let $\psi: M \rightarrow \mathbb{U}_{\mathbb{C}}^{n}(\lambda)$ be an isometric immersion of a real hypersurface in the complex hyperbolic space, such that $\psi(M) \subset \mathcal{H} \mathcal{B}_{\mathbb{C}}$, and let $N$ be the normal vector field of the immersion $\psi$. We will denote by $r$ the distance in $\mathbb{U}_{\mathbb{C}}^{n}(\lambda)$ to the horosphere $\mathcal{H}_{\mathbb{C}}$. If $\partial_{r}$ is the gradient of $r$ in $\mathbb{U}_{\mathbb{C}}^{n}(\lambda)$, and $\partial_{r}^{\top}$ denotes the vector field on $M$ defined from $\partial_{r}$ defined as in the real case, $J \partial_{r}^{\top}(q)$ will denote the vector field on $M$ defined by $J \partial_{r}^{\top}(q)=\psi_{*}^{-1}\left(\mathcal{P}_{q}\left(J \partial_{r}(\psi(q))\right)\right)$, where $J$ denotes the almostcomplex structure in $\mathbb{T}_{\mathbb{C}}^{n}(\lambda)$, and $J \partial_{r}^{J N}(q)$ the vector field $J \partial_{r}^{J N}(q)=$ $\psi_{*}^{-1}\left(\overline{\mathcal{P}_{q}}\left(J \partial_{r}(\psi(q))\right)\right)$, where $\overline{\mathcal{P}_{q}}$ denotes the orthogonal projection $\overline{\mathcal{P}_{q}}: T_{\psi(q)} \mathbb{H}^{n}(\lambda) \rightarrow$ $\{J N\}^{\perp}$ and $\{J N\}^{\perp}$ is the subspace orthogonal to the vector field $J N$ in $\psi_{*} T_{q} M$.

In this case, the $(1,1)$-tensor field $S(r)$ is defined on $\mathbb{U}_{\mathbb{C}}^{n}(\lambda)$ also by $(2.1)$. Since $\psi(M) \subset \mathcal{H} \mathcal{B}_{\mathbb{C}}$, the vector $\partial_{r}$ points inside the horoball bounded by $\mathcal{H}_{\mathbb{C}}$, and $S(r)$ satisfies the following equalities [1]:

$$
\begin{aligned}
S(r) \partial_{r} & =0, \\
S(r)\left(J \partial_{r}\right) & =2 \sqrt{|\lambda|} J \partial_{r}, \\
S(r)(X) & =\sqrt{|\lambda|} X \quad \text { for all } X \in\left\{\partial_{r}, J \partial_{r}\right\}^{\perp}
\end{aligned}
$$

Let $f: \mathbb{R} \rightarrow \mathbb{R}$ be any function and $f(r)$ the composition $f \circ r$. Then, if $\left\{e_{i}\right\}_{i=1}^{2 n-1}$ is a local orthonormal frame of vector fields tangent to $M$, from (2.3) and (3.7) we get

$$
\begin{aligned}
\Delta f(r)= & -\left(f^{\prime \prime}(r)+\sqrt{|\lambda|} f^{\prime}(r)\right)\left\|\partial_{r}^{\top}\right\|^{2}+\sqrt{|\lambda|} f^{\prime}(r)\left\|J \partial_{r}^{\top}\right\|^{2} \\
& +(2 n-1) f^{\prime}(r)\left(\sqrt{|\lambda|}-<H, \partial_{r}>\right) .
\end{aligned}
$$

Now using the function $f(r)=\frac{1}{2 \sqrt{|\lambda|}} e^{-2 \sqrt{|\lambda|}}$, a solution of the differential equation

$$
f^{\prime \prime}(r)+\sqrt{|\lambda|} f^{\prime}(r)=-\sqrt{|\lambda|} f^{\prime}(r)
$$

and noting that

$$
\begin{aligned}
\left\|\partial_{r}^{\top}\right\|^{2}+\left\|J \partial_{r}^{\top}\right\|^{2} & =\left\|\partial_{r}-<\partial_{r}, N>\right\|^{2}+\left\|J \partial_{r}-<J \partial_{r}, N>\right\|^{2} \\
& =1-<\partial_{r}, N>^{2}+1-<J \partial_{r}, N>^{2} \\
& =1+\left\|J \partial_{r}\right\|^{2}-<J \partial_{r}, J N>^{2}-<\partial_{r}, J N>^{2} \\
& =1+\left\|J \partial_{r}-<J \partial_{r}, N>N-<J \partial_{r}, J N>J N\right\|^{2} \\
& =1+\left\|J \partial_{r}^{J N}\right\|^{2} \geq 1,
\end{aligned}
$$

(where we have used the compatibility between $J$ and $<,>$ ), we have

$$
\begin{aligned}
\Delta f(r) & \leq-e^{-2 \sqrt{|\lambda|} r}\left(2 n \sqrt{|\lambda|}-(2 n-1)<H, \partial_{r}>\right) \\
& \leq-(n-1) e^{-\sqrt{|\lambda|} r}(\sqrt{|\lambda|}-\|H\|) .
\end{aligned}
$$

From now on part (i) follows as in the real case.

Part (ii) follows from formula (3.9) as Theorem 2 follows from (3.3). 


\section{REFERENCES}

1. J. Berndt, Real hypersurfaces with constant principal curvatures in complex hyperbolic space, J. Reine Agnew. Math. 395 (1989), 10-19.

2. F. J. Carreras, F. Giménez and V. Miquel, Immersions of compact Riemannian manifolds into a ball of a complex space form, Math. Z. 225 (1997), 103-113.

3. M. do Carmo, Riemannian Geometry (Birkhäuser, Boston, 1992).

4. M. P. Do Carmo and H.B. Lawson, On Alexandrov-Bernstein theorems in hyperbolic space, Duke Math. J. 50 (1983), 995-1003.

5. J. Miranda de Gomes, Spherical surfaces with constant mean curvature in the hyperbolic space, Bol. Soc. Brasil Mat. 18 (1987), 49-73.

6. Th. Hasanis and D. Koutroufiotis, Immersions of Riemannian manifolds into cylinders, Arch. Math. (Basel) 40 (1983), 82-85.

7. D. Hoffman and W. H. Meeks, The strong halfspace theorem for minimal surfaces, Invent. Math. 101 (1990), 373-377.

8. D. Hoffman and W. H. Meeks, Embedded minimal surfaces of finite topology, Ann. of Math. (2) 131 (1990), 1-34.

9. L. Jorge and D. Koutroufiotis, An estimate for the curvature of bounded submanifolds, Amer. J. Math. 103 (1981), 711-725.

10. L. Jorge and F. Xavier, A complete minimal surface in $\mathbb{R}^{3}$ between two parallel planes, Ann. Math. 112 (1980), 203-206.

11. L. Jorge and F. Xavier, An inequality between the exterior diameter and the mean curvature of bounded immersions, Math. Z. 178 (1981), 72-82.

12. F. López and F. Martín, Complete nonorientable surfaces and symmetries, Duke Math. J. 79 (1995), 667-686.

13. H. Omori, Isometric immersions of Riemannian manifolds, J. Math. Soc. Japan 19 (1967), 205-214. 